\title{
Partial discharge investigation on palm oil using needle - plane electrode configuration and electric field distribution using ANSYS Maxwell
}

\begin{abstract}
The Partial Discharge Inception Voltage (PDIV) and PD characteristic of mineral oil were described and compared with palm oil. The test was performed by needle-plane electrode configurations. Both mineral- and palm oil were investigated under AC voltage. A comparison study between PDIV and PD activities of the mineral oil Hyrax and palm oil were investigated with the total volume of the oil in the test cell was approximately $2 \mathrm{~L}$. The $50-\varepsilon m$ tip radius of tungsten needle electrodes were utilized as high voltage electrode. On the other hand, the grounded electrode with 30-, 40-, and $50 \mathrm{~mm}$ gap distances was represented by a $50-\mathrm{mm}$ and $75-\mathrm{mm}$ diameter copper plane electrode. Consideration on the oil conditions' effect on both PDIV and PD characteristics was also carried out. It was clearly shown that PDIV is dependent on the electric field stress of the electrode system and the test method based on the experimental results. In comparison to differences of gap distance, the $50-\varepsilon m$ needle tip radius and 75-mm plane with gap distance of 30-mm electrode revealed the highest electric field stress and inversely exhibited the lowest PDIV value tested. Furthermore, simulation using ANSYS Maxwell on the electric field distributions of the electrode systems was also performed. In order to quantify electric field based on the finite element methods the ANSYS Maxwell was being utilized. In the simulation, the palm oil and mineral oil properties of its 2D modelling test cell was used. From the results, the PD characteristics of mineral oil and palm oil tested by needle-plane electrode system are known. To confirm the suitability of palm oil as a high voltage insulating medium, it is vital to analyse in-depth on its partial discharge activity and characteristics.
\end{abstract}

Keyword: Partial discharge; PDIV; Mineral oil; Palm oil; Electric field; ANSYS 